\title{
Evolution of the plasmoid-lobe interaction with downtail distance
}

\author{
J.A. Slavin, C.J. Owen and M. Hesse \\ Laboratory for Extraterrestrial Physics, NASA/Goddard Space Flight Center, Greenbelt, Maryland
}

Abstract. This study examines the interaction between plasmoids moving anti-sunward at high speeds and the tail lobes which bound them to the north and south. Attention is focused on the influence of changing lobe conditions with downtail distance. It is shown using ISEE 3 measurements that the gradual filling of the lobes with mantle plasma and the decrease in magnetic field intensity reduces the average lobe MHD fast mode speed from $1200 \mathrm{~km} \mathrm{~s}^{-1}$ at $X=-80 R_{E}$ to $400 \mathrm{~km} \mathrm{~s}^{-1}$ at $X=-220$ $R_{E^{*}}$. This results in the ratio of the plasmoid speed to the fast mode speed increasing with downtail distance, from 0.3 at $\mathrm{X}=$ $-80 R_{E}$ to $\sim 1$ at $X=-220 R_{E}$. It is argued that the "standard" traveling compression region (TCR) signature observed closer to the Earth will be distorted at large distances, where the fast mode transit time between the plasmoid and magnetopause becomes long compared to the time for the plasmoid to move past a given point in the tail. This change in the nature of the plasmoid-lobe interaction with downtail distance is offered as an explanation for why the reported rate of TCR occurrence peaks at $X=-60$ to $-130 R_{E}$ and decreases in the more distant tail.

\section{Introduction}

Traveling compression regions are several-minute long enhancements of the magnetic field intensity in the lobes of the tail, accompanied by a north-then-south variation in the $\mathrm{B}_{z}$ component [Slavin et al., 1984]. Further studies have supported the original interpretation that these compressive perturbations in the lobe field are caused by the rapid tailward movement of plasmoids [Owen and Slavin, 1992; Slavin et al., 1993; Nagai et al., 1994]. According to this model, the greater north-south dimensions of the plasmoid relative to the plasma sheet result in the lobe field lines being compressed and draped about the plasmoid. This local compression of the lobe magnetic field then accompanies the plasmoid as it moves down the tail and can be observed by spacecraft residing in the lobes. In this paper, the plasmoid-lobe-magnetosheath interaction is examined in more detail with an emphasis on the effects of changing lobe conditions with increasing downtail distance. It is found that the TCR signature frequently observed at distances of $X=-60$ to $-130 R_{E}$ may be significantly distorted in the distant tail due to the decrease in the fast mode speed as the lobe fills with mantle plasma. The magnetopause response to the TCR, the transmission ratio for this wave across the lobe-magnetosheath interface, and the "wave drag" exerted on plasmoids are also discussed.

\section{Traveling Compression Regions}

An example of a typical TCR observed by ISEE 3 is displayed in Figure 1. Plasma analyzer measurements clearly indiCopyright 1994 by the American Geophysical Union.

Paper number 94GL02442

0094-8534/94/94GL-02442\$03.00

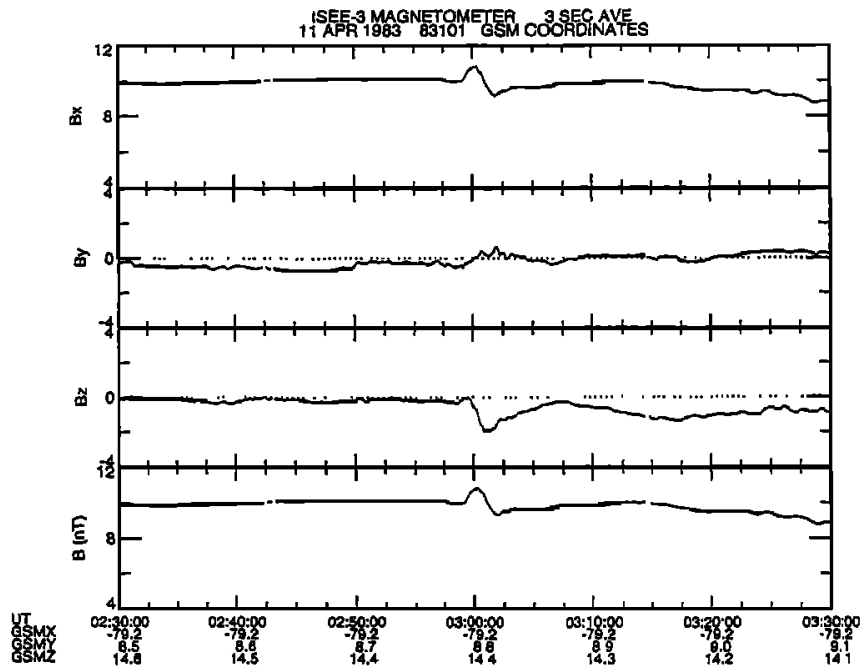

Figure 1. A typical example of a TCR at $X=-79 R_{E}$ observed by ISEE 3. Note the nearly bell-shaped compression and the sinusoidal $B_{Z}$ variation.

cate that ISEE 3 was in the north tail lobe throughout this interval [e.g., Zwickl et al., 1984]. The TCR is identified by the several-minute long compression in the field magnitude centered on 03:00:20 UT, the bipolar variation in $\mathrm{B}_{z}$ with the inflection point coincident with the field maximum, and the extended interval of $\mathrm{B}_{\mathrm{z}}<0$ afterwards [Slavin et al., 1984; 1993]. The bipolar variation in $B_{z}$ is the signature of the lobe field draping over the plasmoid in the $X-Z$ plane. The extended interval of southward $\mathrm{B}_{\mathrm{Z}}$ following the bipolar signature is caused by the reconnection of lobe field lines following the plasmoid release.

Slavin et al. [1993] found the occurrence rate of TCRs is not constant as a function of downtail distance. The number of TCRs per unit time increases until it reaches a broad maximum at $X=-60$ to $-130 R_{E}$. The gradual increase is consistent with plasmoids typically having lengths of 10 to $60 \mathrm{R}_{\mathrm{E}}$ [Richardson et al., 1987; Moldwin and Hughes, 1992] and forming Earthward of $X=-100 R_{E}$. However, the observed TCR frequency then falls by about a factor of 5 by $X=-200 R_{E}$. No corresponding decrease in the plasmoid occurrence rate has been observed [Moldwin and Hughes, 1992]. Some of this decrease may be due to the obscuring of TCR signatures by enhanced wave activity in the plasma sheet boundary layer at these distances. However, the overall decrease in the numbers of TCRs at large downtail distances has never been satisfactorily explained.

\section{Fast Mode Speed in the Lobes}

As a plasmoid moves downtail it must launch fast mode waves (primarily in the $+/-\mathrm{Z}$ directions) to compress and displace the lobe flux tubes in its path. Accordingly, the ratio of 


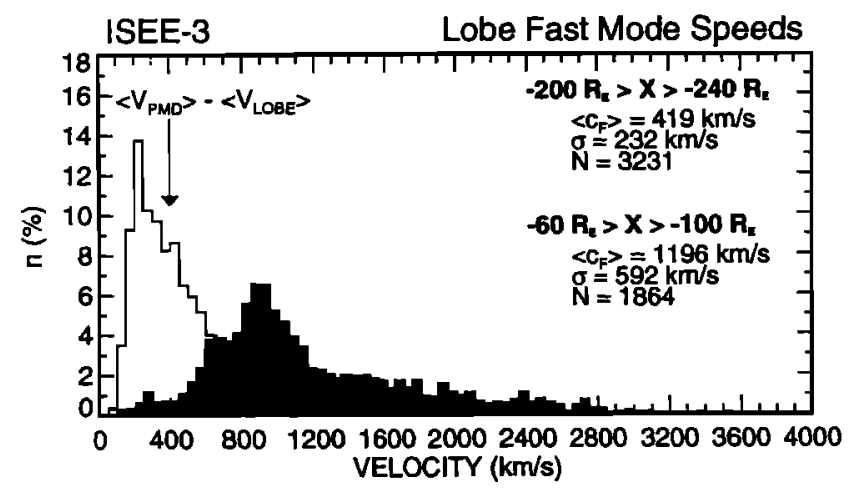

Figure 2. Histograms of ISEE 3 5-min averaged MHD fast mode speed at two different downtail distances. The mean plasmoid speed in the rest frame of the tailward moving lobe plasma is also indicated.

the plasmoid speed to the lobe fast mode speed is a critical parameter in determining how the lobe fields drape about plasmoids and the nature of the TCR. Plasmoid studies utilizing the ISEE 3 plasma measurements have reported plasmoid speeds from $\sim 300$ to $1200 \mathrm{~km} \mathrm{~s}^{-1}$ with a mean near $600 \mathrm{~km} \mathrm{~s}^{-1}$ [Richardson et al., 1987; Moldwin and Hughes, 1992].

Figure 2 shows fast mode speeds measured when ISEE 3 was located in the tail lobes at distances of $X=-60$ to $-100 R_{E}$ (darker shaded histogram) and -200 to $-238 R_{E}$ (lighter histogram). As thermal ions were not measured, it is assumed that $\mathbf{n}_{1}$ $=n_{c}$ and $T_{i}=T_{e}$. The latter assumption is not critical as the lobe plasma is very cold and the sound speed is insignificant relative to the Alfven speed. To include a mean contribution from heavier ions to the fast mode speed, the lobe composition is assumed to be $95 \% \mathrm{H}^{+}$and $5 \% \mathrm{O}^{+}$[e.g., Chappell et al., 1987].

In general, the fast mode speed is expected to decrease with distance downtail as mantle plasma fills the lobes and the field strength drops due to reduced magnetopause flaring [Zwickl et al., 1984; Slavin et al., 1985; Yamamoto et al., 1994]. The results in Figure 2 confirm this expectation. At $X=-60$ to -100 $R_{E}$, the mean lobe fast mode speed is $\sim 1200 \mathrm{~km} \mathrm{~s}^{-1}$. At $X=-200$ to $-238 R_{E}$ the average fast mode speed has fallen to $\sim 400$ $\mathrm{km} \mathrm{s}^{-1}$. For comparison, the typical plasmoid speed is 600 $\mathrm{km} \mathrm{s}^{-1}$ and the average flow speed of the mantle plasma in the lobes is about $200 \mathrm{~km} \mathrm{~s}^{-1}$ tailward [Zwickl et al., 1984]. Thus the average plasmoid speed relative to the lobe plasma is $\sim 400$ $\mathrm{km} \mathrm{s}^{-1}$ as indicated by the arrow in Figure 2. Accordingly, the ratio of the mean plasmoid speed (in the lobe plasma rest frame) to the lobe fast mode speed increases from $\sim 0.3$ to 1 between $X=-80$ and $-220 R_{E}$. If the most common fast mode speeds are used, i.e., the modes of the distributions, the change in this ratio is even more dramatic, 0.4 to 2 .

\section{Plasmoid-Lobe Interaction}

Figure 3 displays schematically the interaction between the tailward-moving plasmoid and the lobes. For the sake of illustration, the presence of the plasma sheet in front of and behind the plasmoid is neglected and the plasmoid length and the lobe width are assumed equal. In the top panel, Figure 3a, the plasmoid speed is taken to be less than the fast mode speed as is typical for $X>-150 R_{E^{*}}$. In this case, there is ample time for the fast mode compression and rarefaction waves launched by the plasmoid (indicated by dark arrows) to drape the lobe flux tubes closely about the plasmoid and produce the clean bipolar $B_{z}$ signature usually associated with TCRs (e.g., see Figure 1). A sample field line has been drawn to show the draping pattern. Farther out in the lobes the TCR lags slightly behind the plasmoid and the magnetopause bulges outward slightly in response to the pulse of enhanced pressure. Theoretical models of the field line draping in this low Mach number regime have been developed by Birn [1992] which support this picture.

In Figure $3 \mathrm{a}$ the fast mode transit time between the plasmoid and the magnetopause is less than the time for the plasmoid to move past a given point in the tail. Hence, the gradients in the $\mathrm{Z}$ direction are small and a balance exists between the plasma and magnetic pressures in the plasmoid, the magnetic pressure in the lobes, and external magnetic and plasma pressures in the magnetosheath, including any ram pressure associated with the local bulging of the magnetopause. This is the simple TCR picture assumed by previous studies [e.g., Slavin et al., 1984].

The case where the plasmoid moves at twice the fast mode speed is treated in Figure $3 \mathrm{~b}$. In this situation, the lobe compression region will lag significantly behind the plasmoid. The fast mode transit time between the plasmoid and the magnetopause will be much less than the plasmoid transit time past a given point in the tail. Hence pressure equilibrium does not exist in planes perpendicular to the $\mathrm{X}$ axis. The interaction now resembles that of the bow wave produced by a boat in a channel.

Also addressed in Figure $3 b$ is the effect the higher ratio of plasmoid to fast mode speed has on the draping pattern of the lobe fields over the plasmoid. On the anti-sunward side of the plasmoid, treating the lobes as an MHD fluid leads to the expectation that the lobe flux tubes will be more compressed than in the $\mathrm{V}_{\mathrm{PMD}} / \mathrm{C}_{\mathrm{F}}<1$ case and the leading edge of the compression region may exhibit some nonlinear steepening. In contrast, the trailing portion of the TCR will be greatly broadened because the plasmoid is moving downtail much faster than the fast mode
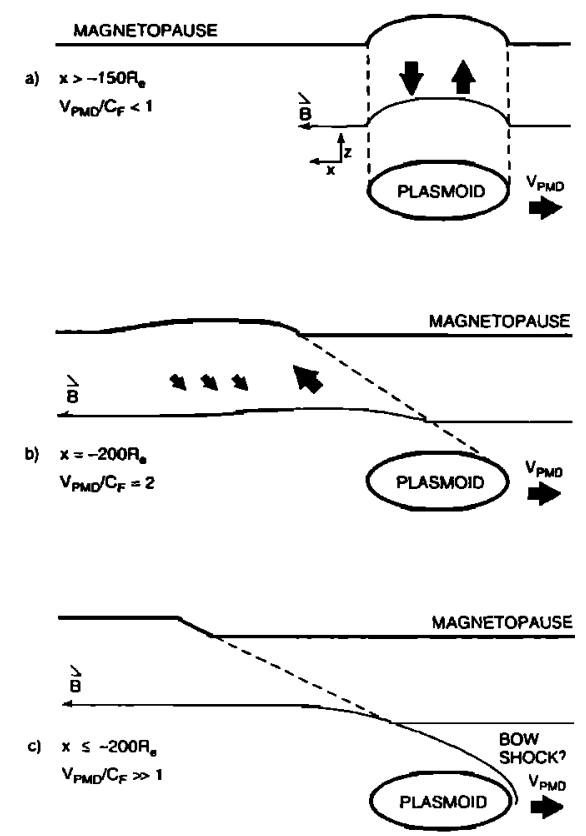

Figure 3. Idealized views of the plasmoid-lobe interaction for three different $V_{P M D} / C_{F}$ ratios are displayed. Note the change in the field line draping about the plasmoid as the ratio of its tailward speed to the fast mode speed in the lobes increases. 
rarefaction wave can fill-in behind the plasmoid and return the lobe field lines to their equilibrium positions. This asymmetry will also be seen at the magnetopause which will take longer to return to its original location. Hence, for $V_{P M D} / C_{F}>1$ both the TCR pressure pulse and its bipolar $B_{z}$ may become highly asymmetric with steepening on the leading side and shallower, longer duration signatures on the trailing side. This may result in the plasmoid-associated field line draping and compression signatures diverging from the standard symmetric signatures to the point where the criteria used to identify TCRs in previous studies [e.g., Slavin et al., 1984; 1993] are no longer satisfied.

Finally, it is interesting to speculate about the extreme case in which the plasmoid moves downtail at much greater speed than that of fast mode waves in the lobes. The prima facia interpretation of Figure 2, for example, suggests that $\mathrm{V}_{\mathrm{PMD}} / \mathrm{C}_{\mathrm{F}}$ may sometimes be as great as 7 . Under such conditions the Mach cone for the pressure pulse radiated by the plasmoid will become smaller and the lobe compression and the magnetopause bulge will lag still farther behind the plasmoid, as shown in Figure $3 \mathrm{c}$. Depending upon the ratio of the plasmoid height in $\mathrm{Z}$ to its length in $X$, the anti-sunward side of the plasmoid may be locally moving up into the lobes at super-fast mode speeds. In this case, the leading edge of the TCR may stand-off and steepen into a fast mode shock. Earthward of the plasmoid it will probably decay into a bow wave as shown by the dashed line in Figure 3c. Whether or not a shock forms in the low plasma beta environment of the lobes is a complex problem which will need careful examination before being accepted as a real possibility. Nevertheless, the standard TCR signature is expected to become increasingly distorted and asymmetric as $\mathrm{V}_{\mathrm{PMD}} / \mathrm{C}_{\mathrm{F}}$ increases.

\section{Magnetopause Response}

Another aspect of the plasmoid-lobe-magnetosheath interaction is the degree to which the magnetopause bulges outward in response to the plasmoid passage. A significant increase in tail diameter is often assumed when depicting the large scale configuration changes of the distant tail during substorms. The amplitude of the magnetopause bulge is physically important in that it will determine the magnitude of any ram force exerted by the net speed difference between the magnetosheath plasma and the bulge. A better understanding of the magnetopause response to the passage of plasmoids is essential to a more complete description of the TCR phenomenon and distant tail dynamics.

Recently, Slavin et al. $[1989 ; 1993]$ investigated the dimensions of plasmoids and their impact on magnetopause diameter. The first study, analyzing CDAW 8 plasmoids, found that the magnitude of the internal $B_{Z}$ fields were only about twice those typical of the plasma sheet at the distances where plasmoids form. If the plasma in this region is relatively incompressible, as is generally believed, then the north-south dimensions of plasmoids are only twice that of the quiescent plasma sheet. Fairfield [1992] statistically determined the latter to be $7 \mathrm{R}_{\mathrm{E}}$, such that the mean north-south plasmoid height is then $\sim 14 R_{E}$.

Slavin et al. [1993] considered the local effects of requiring the magnetopause to expand significantly on the several minute time scale of TCRs. They noted that increases of more than -1 $R_{E}$ require the magnetopause to expand into the magnetosheath at super-fast mode speeds. This, in turn, implies extremely large lobe magnetic fields to balance the ram forces experienced as the external solar wind plasma is pushed outward. The small amplitude of the compression, averaging $\sim \mathrm{nT}$ [Slavin et al., 1993], led them to conclude that the magnetopause does not ex- pand outward at velocities greater than the magnetosheath fast mode speed, typically $70 \mathrm{~km} \mathrm{~s}^{-1}$. Given the 3 min mean duration of TCRs (N.B., the magnetopause moves outward only during the rising half of the pressure pulse), the magnetopause is expected to move outward by $\sim 1 R_{E}$ during these events.

Finally, it is of interest to examine the effect of this bulge in the magnetopause on pressure balance with the magnetosheath. A $1 R_{E}$ increase in tail diameter over a distance of half a typical plasmoid length, about $15 R_{E}$, corresponds to a flaring angle of $4^{\circ}$. If the magnetosheath plasma is flowing at $400 \mathrm{~km} \mathrm{~s}^{-1}$ and the TCR is traveling at $600 \mathrm{~km} \mathrm{~s}^{-1}$, then the net speed difference is $200 \mathrm{~km} \mathrm{~s}^{-1}$. For a mean distant magnetosheath density of $6 \mathrm{~cm}^{-3}$, the dynamic pressure normal to the bulge under the Newtonian approximation with $4^{\circ}$ of flaring is $2 \times 10^{-11}$ dynes $\mathrm{cm}^{-2}$. The increase in the lobe field necessary to balance such a pressure is $\sim 2 \mathrm{nT}$. The fact that this enhancement is similar to, but larger than the $1 \mathrm{nT}$ typical of TCRs [Slavin et al., 1993], supports the previous arguments that the magnetopause probably moves outward by no more than $1 \mathrm{R}_{\mathrm{E}}$ in response to the pressure pulse launched by plasmoids moving down the tail. Hence, it is concluded that the magnetopause behaves as a quasi-rigid boundary with respect to the downstream movement of plasmoids.

\section{Wave Transmission at the Magnetopause}

Having determined the magnetopause moves only slightly outward in response to the TCR pressure pulse, one may ask what fraction of the power in the compression wave is transmitted across the magnetopause and what fraction is reflected. Treating this fast mode wave front as propagating exactly perpendicular to the lobe field, the impedance of a medium of mass density $\rho$ is, by analogy to simple acoustic waves, simply:

$$
Z=C_{F} p
$$

The reflection coefficient is then

$$
R_{12}=\left(Z_{1}-Z_{2}\right) /\left(Z_{1}+Z_{2}\right)
$$

Using ISEE 3 measurements, the mean characteristic impedance of the lobe at $-60 R_{E}>X>-100 R_{E}$ is $Z_{1}=1.4 \times 10^{-16} \mathrm{~kg} \mathrm{~m}^{-2} \mathrm{~s}^{-1}$ and $2.4 \times 10^{-16} \mathrm{~kg} \mathrm{~m}^{-2} \mathrm{~s}^{-1}$ at $-200 R_{E}>\mathrm{X}>-240 \mathrm{R}_{E}$. Typical distant magnetosheath fast mode speeds and densities are essentially those of the solar wind at $1 \mathrm{AU}, 70 \mathrm{~km} \mathrm{~s}^{-1}$ and $6 \mathrm{~cm}^{-3}$, respectively, so $Z_{2}=7 \times 10^{-16} \mathrm{~kg} \mathrm{~m}^{-2} \mathrm{~s}^{-1}$. With this relatively close match between impedances, the reflection coefficient is small and the fraction of wave power transmitted, $1-\mathrm{R}^{2}$, is $56 \%$ and $76 \%$ in the two distance regimes, respectively. Most of the incident energy carried by the wave front is thus transmitted across the magnetopause and goes into compressing and heating the external magnetosheath. As downtail distance increases, the magnetopause becomes more transparent to the compression wave fronts radiated by the tailward-moving plasmoids.

\section{Wave Drag on the Plasmoid}

The energy flux carried by the TCR is derived from the plasmoid kinetic energy and represents a drag force. In fluid mechanics, energy loss associated with compression waves which displace fluid parcels in a projectiles path is generally termed "pressure drag", or in supersonic flows, "wave drag". The TCR energy flux represents a Poynting flux which can be calculated from the fast mode speed and the compression amplitude

$$
\mathrm{S}=(1 / 8 \pi) \Delta \mathrm{B}_{\mathrm{TCR}}^{2} \mathrm{C}_{\mathrm{F}}
$$


where $\mathrm{C}_{\mathrm{F}}=1200 \mathrm{~km} \mathrm{~s}^{-1}$ and $\left.\angle \Delta \mathrm{B}_{\mathrm{TCR}}\right\rangle=0.5 \mathrm{nT}$ at $\mathrm{X}=-60$ to $-100 \mathrm{R}_{\mathrm{E}}$. The corresponding energy flux is $10^{-4} \mathrm{ergs} \mathrm{cm}^{-2} \mathrm{~s}^{-1}$. In the $V_{P M D} / C_{F}<1$ regime, where the TCR amplitude is constant between the plasmoid and the magnetopause (e.g., Figure 3a), this Poynting flux passes through an annulus with the mean radius of the tail, $24 R_{E}$, and the typical plasmoid length, $30 R_{E}$, corresponding to an area of $2 \times 10^{21} \mathrm{~cm}^{2}$. Assuming half of the wave energy is transmitted across the magnetopause, the energy loss experienced by an average plasmoid is $10^{10}$ Joules $\mathrm{s}^{-1}$.

Given that the total kinetic energy of a typical plasmoid has been estimated to be about $10^{14}$ Joules [Slavin et al., 1989; 1993], wave drag alone would require $\sim 10^{4} \mathrm{~s}$, or $\sim 3 \mathrm{hrs}$, to bring a plasmoid to rest. The energy loss due to wave drag is, therefore, much smaller in magnitude than the tailward pressure gradients and $\mathbf{J} \times \mathbf{B}$ forces which rapidly accelerate the plasmoid at the time of its release [see Hesse and Birn, 1991]. However, wave drag may still be important in determining the final, or terminal speed of plasmoids. For example, the sunward wave drag force on the plasmoid may be approximately balanced by the residual anti-sunward $\mathbf{J} \times \mathbf{B}$ force due to the disconnected lobe field lines draped about the trailing end of the plasmoid.

\section{Summary}

Analysis of ISEE 3 observations indicates that the mean ratio of plasmoid speed to the lobe fast mode speed increases from 0.3 at $X=-80 R_{E}$ to 1 at $X=-220 R_{E}$. Using simple MHD wave propagation arguments it is shown that the decrease in fast mode speed will affect the draping of lobe field lines about the plasmoid and alter the simple TCR signature observed closer to the Earth where typically $V_{P M D} / C_{F}<1$. In particular, the relatively symmetric bipolar $B_{Z}$ and normal-shaped field intensity increase used to identify TCRs may become rare. Instead, the compressive perturbation caused by the plasmoids will become increasingly asymmetric with a steepening of the leading edge of the initial northward $B_{Z}$ and field magnitude increase. Conversely, the trailing southward $B_{2}$ and field intensity decrease will take place over an extended interval of time. In extreme cases the leading edge of the TCR near the plasmoid may even steepen into a fast mode shock wave. Thus the usually symmetric and readily identifiable TCR signature seen closer to the Earth may be distorted to the point where only compression regions associated with slow moving plasmoids (or times when the lobe fast mode speed is higher than average) will be identified as such. If correct, this new picture of the change in plasmoid-lobe interaction with downtail distance suggests that the utility of TCRs as plasmoid indicators may degrade beyond $X \sim-150 R_{E}$. This is a promising explanation for the puzzling decrease in the TCR $\propto$ currence rate in the distant tail observed by Slavin et al. [1993].

Finally, most of the compressional wave energy associated with TCRs is transmitted across the magnetopause and lost to the magnetosheath. This energy constitutes a drag force which was estimated using simple Poynting flux arguments. This MHD analogue of "wave drag" is too small to greatly slow the plasmoid inside of the ISEE 3 and Geotail apogees. However, wave drag may play some role in setting the "terminal speed" of plasmoids as they go downtail. At this juncture the forces acting on plasmoids are too poorly known for this to be determined.

Acknowledgments. The authors acknowledge the use of ISEE 3 magnetometer (P.I. - E. J. Smith) and plasma analyzer (P.I.- S.J. Bame) measurements. Extremely useful discussions with M.J. Lyell on pressure and wave drag in fluid media are gratefully acknowledged. JAS wishes to thank $\mathrm{H}$. Koskinen for pointing out an arithmetic error in the plasmoid energy estimate in Slavin et al. (1993). Comments and suggestions by J. Birn, D. Fairfield, M. Kuznetsova, M. Moldwin, D. Sibeck and S. Taguchi are also acknowledged.

\section{References}

Birn, J., Quasi-steady current sheet structures with field-aligned flow, J. Geophys. Res., 97, 16,817, 1992.

Chappell, C.R., et al., The ionosphere as a fully adequate source of plasma for the Earth's magnetosphere, J. Geophys. Res., 92,5896, 1987.

Hesse, M., and J. Birn, Plasmoid evolution in an extended magnetotail, J. Geophys. Res., 9I,5683, 1991.

Fairfield, D.H., On the structure of the distant magnetotail: ISEE 3, J. Geophys. Res., 97, 1,403, 1992.

Moldwin, M.B., and W.J. Hughes, On the formation and evolution of plasmoids: A survey of ISEE 3 geotail data, J. Geophys. Res., 97, $19,259,1992$.

Nagai, T., et al., Initial Geotail survey of magnetic substorm signatures in the magnetotail, In Press, Geophys. Res. Lett., 1994.

Owen, C.J., and J.A. Slavin, Energetic ion events associated with traveling compression regions, Proc. International Conf. on Substorms, ESA SP-335, pp. 365-370, May, 1992.

Richardson, I.G., et al., Plasmoid-associated energetic ion bursts in the deep geomagnetic tait: Properties of plasmoids and the postplasmoid plasma sheet, J. Geophy.s. Res., 92, 9,997, 1987.

Slavin, J.A., et al., Substorm-associated travelıng compression regions in the distant tail: ISEE 3 gcotail observations, Geophys. Res. Lett, $11,657,1984$.

Slavin, J.A., et al., An ISEE 3 study of average and substorm conditions in the distant magnetotail, J. Geophys. Res., s' 10,875, 1985.

Slavin, J.A., et al., CDAW 8 observations of plasmoid sıgnatures in the geomagnetic tail: An assessment, J. Geophy.s. Res., 94, 15,153, 1989.

Slavin, J.A., et al., ISEE 3 observations of traveling compression regions in the Earth's magnetotail, J. Geophys. Res., 98, 15,425, 1993.

Yamamoto, $\mathbf{T}$., et al., Dense plasmas in the distant magnetotail as observed by Geotail, In Press, Geophys. Res. Lett., 1994.

Zwickl, R.D., et al., Evolution of the Earth's distant magnetotail: ISEE 3 electron plasma results, J. Geophys. Res., 89, 11,007, 1984.

J.A. Slavin, C.J. Owen and M. Hesse, Laboratory for Extraterrestrial Physics, NASA/Goddard Space Flight Center, Greenbelt, MD 20771. (e-mail: slavin@lepjas.gsfc.nasa.gov)

(Received: June 22, 1994; accepted: August 17, 1994) 